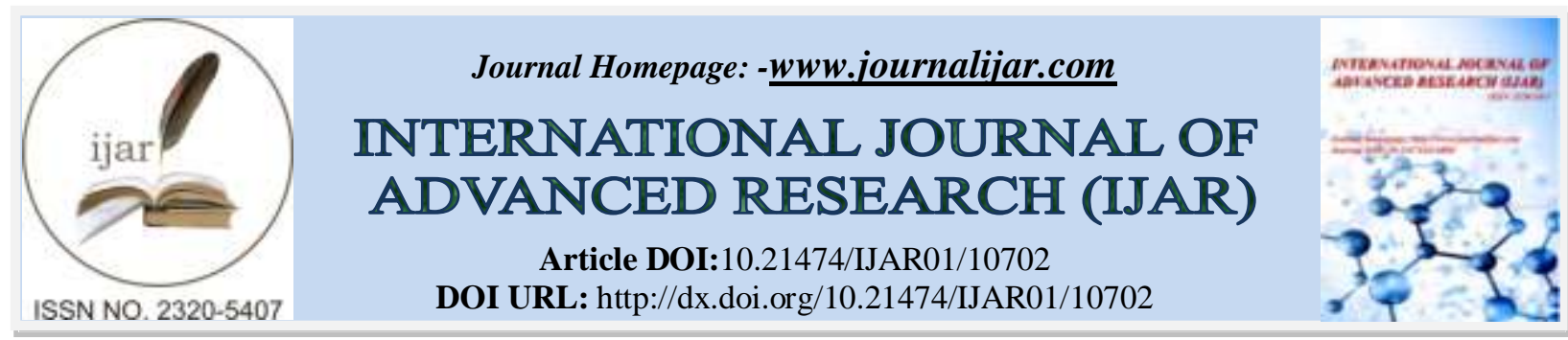

RESEARCH ARTICLE

\title{
INDUSTRIAL ZOO/BIOPLANKTON CULTIVATION IN THE OCEAN FOR AGRICULTURE AND MEDICINE NEEDS
}

\author{
A.V. Kulakov and V.A. Rantsev-Kartinov
}

Institute of Functional Economic Systems, Moscow, Russia.

Manuscript Info
Manuscript History
Received: 20 January 2020
Final Accepted: 22 February 2020
Published: March 2020
Key words:-
$\begin{aligned} & \text { Universal Modules of Industrial } \\ & \text { Disintegrators/Activators (UMoID/A), } \\ & \text { Nanocement/Concrete, Nanoparticles } \\ & \text { from Various Chemical Elements }\end{aligned}$

\section{Manuscript Info}

Manuscript History

Final Accepted: 22 February 2020

Key words:-

Disintegrators/Activators (UMoID/A),

from Various Chemical Elements

\begin{abstract}
UMoID/A, developed by the authors of this work, have unique performance specifications (extremely high magnetic induction density, close to one hundred percent efficiency, low material consumption), which allows them to grind various materials up to nanoscale for various areas of industrial production, chemistry and medicine. These unique specifications of UMoID/A allowed us to use these disintegrators in our developed technologies for producing cheap alkaline nanocement/concrete, cheap and eco-friendly cement wood, cheap carbon nanotubes, cheap combined feed, increasing the ultimate production of light oil fraction under the subsequent standard process of its cracking, as mixers for producing high-quality suspensions in the oil, coal and construction industries. The main attention in this work is devoted to solving the most important global food and environmental problem - the industrial zoo/bioplankton cultivation in the ocean based on our developed technology for producing cheap nanoparticles from various chemical elements, as well as multilayer telescopically embedded carbon nanotubes by grinding modified coal using the UMoID/A. This project will allow to comprehensively solve the fundamental problems of obtaining renewable energy sources for obtaining products for agriculture and medicine needs, as well as to solve the problem of global warming by consuming a large amount of carbon dioxide dissolved in ocean water and accumulated in the atmosphere for this project. All the technologies proposed in this article by the authors are based on the use of electromagnetic devices designed and patented by us with a vortex ferromagnetic layer $[1,2]$. Due to the versatility of their highly efficient application in various areas of industrial production, they are called as "Universal Modules of Industrial Disintegrators/Activators" UMoID/A.
\end{abstract}

\section{Introduction:-}

The UMoID/A versatility and effectiveness is due to:

A variety and activity of processes occurring in its working area:

1. "constrained blow" crushing;

2. electromagnetic erosion;

3. plasma-basedexposure; 
4. ultrasound (when processed in a liquid medium);

1. high energy density of magnetic induction in its working area (at $\mathrm{B} \sim 10^{4} \mathrm{Gs}, \mathrm{W} \sim 0.4 \mathrm{~J} / \mathrm{cm}^{3}=4105 \mathrm{~J} / \mathrm{m}^{3}$ ), which is hundreds of times higher than the energy density in the working areas of other similar devices;

2. where $\mathrm{B}$ is the magnetic field strength, $\mathrm{W}$ is the magnetic energy density

3. close to one hundred percent efficiency;

4. low material consumption;

5. highreliability.

We list some of the capabilities of UMoID/A and the following proposals of new technologies that show what unique applications the UMoID/A have if the authors fully understand the physical processes occurring in the Multifunction Modules:

The UMoID/A is a highly efficient mill that allows to grind various materials up to nanoscale for the needs of various fields of industrial production, chemistry and medicine. At this production achievement, the UMoID/A made it possible to develop effective production technologies:

cheap alkaline cement/concrete (made of rocks and volcanic rocks without their annealing, but with small corrective additives and electric current passing through the concrete mass) with a strength of up to $\sim 2000 \mathrm{~kg} / \mathrm{cm}^{2}, \mathrm{resistant}$ in sea water;

cheap and eco-friendly wood concrete from such an alkaline cement filled with wood chips, sawdust, reeds, straw and with a strength of up to $\sim 300 \mathrm{~kg} / \mathrm{cm}^{2}$, thermal conductivity like wood and a specific gravity of $\sim 1.3 \mathrm{~g} / \mathrm{cm}^{3}$;

cheap combined feed containing the same amount of feed units as grain, by processing grain straw, rough grass and with a $30 \%$ addition of grain flour;

cheap nanoparticles from various chemical elements, as well as multilayer, telescopically embedded carbon nanotubes by grinding modified (according to the technology developed by the authors) coal for the needs of various industries[3].

obtaining $56.3 \%$ of starch from potatoes, which is 2.5 times higher than the well-known level of -22\%. [4]

The knowledge set forth in paragraph " $d$ " allowed the authors of the article to formulate new cheap technologies for industrial:

production of new composite nanomaterials from carbon nanotubes for the needs of various areas of industrial production, chemistry and medicine;

zooplankton cultivation in the ocean for agriculture and medicine needs.

The UMoID/A can be used as a highly efficient oil pre-treatment device before the standard cracking process, which allows increasing (up to 5\%) the ultimate production of light oil fraction during the subsequent standard process of its cracking.

The UMoID/A is a highly efficient mixer, which makes it possible to obtain high-quality, uniform suspensions of such substances as:

heavy still bottoms/coal dust with water or with diesel fuel to produce cheap boiler or diesel fuel from urban thermal power plants or powerful ship/pit-run diesel engines;

oil with chalk stone or clay as stable non-settling drilling fluids for a long time.

The UMoIDA is used as a highly effective activator and accelerator of physical \chemical processes in various fields of industrial production of new types of building materials, porcelain, delftware, ceramics, paints, perfume 
composites, nanocomposite materials (the reaction intensity in the UMM working area is thousands of times higher than in others types of reactors).

The grinding in the UMoID/A is carried out mainly under the influence of the so-called "constrained blow" implemented under a turbulent ferromagnetic layer of the working fluid particles environment under the influence of an electromagnetic field traveling in the working area. As a result of the "constrained blow", the grains of obtained powders have very sharp edges, which contribute to an increase in both their physical and chemical activity. This makes it possible to obtain greater activity of the "binder" produced from them during "pre-mixing" with an alkali or soda solution and higher activity when used in powder chemistry. This characteristic also contributes to the suspensions preparation from these powders process activation. Due to the powerful turbulent movement of the mixture of liquids in the working area, the UMoID/A can be effectively used to prepare emulsions of practically immiscible liquids. The processes of materials grinding in the working area of the module are superficial, therefore, these processes become more active when the initial product specific area become higher. Under the conditions of material processing processes in the UMoID/A, no "balling up" of powders occurs, therefore, with an increase in the powders fineness, the process efficiency does not decrease, but rather increases. Since there are no bearings or rubbing structural elements in the working area of the module, during device operation, in principle, jamming and equipment shutdown cannot occur. This equipment is low-noise with low material consumption compared to other similar equipment for this purpose. Only walls that limit the sleeve working areas and the working fluid elements, which are easily replaced during equipment routine maintenance, are subject to wear. The durability of the working area sleeve made of X18H9T alloy is $\sim 1000$ hours.

The technical and economic advantages of the UMoID/A in comparison with the tubular ball mills reference specimen are:

1. 30-50\% reduction in specific electricity consumption;

2. lack of high-wearing grinding media and plates;

3. reliability indicators increase;

4. decrease in specific metal consumption and space dimensions;

5. noise reduction.

To obtain the desired effect during each technology development, the UMoID/A operating modes and material holding in its working area time are selected.

The UMoID/A design is schematically shown in Fig. 1.

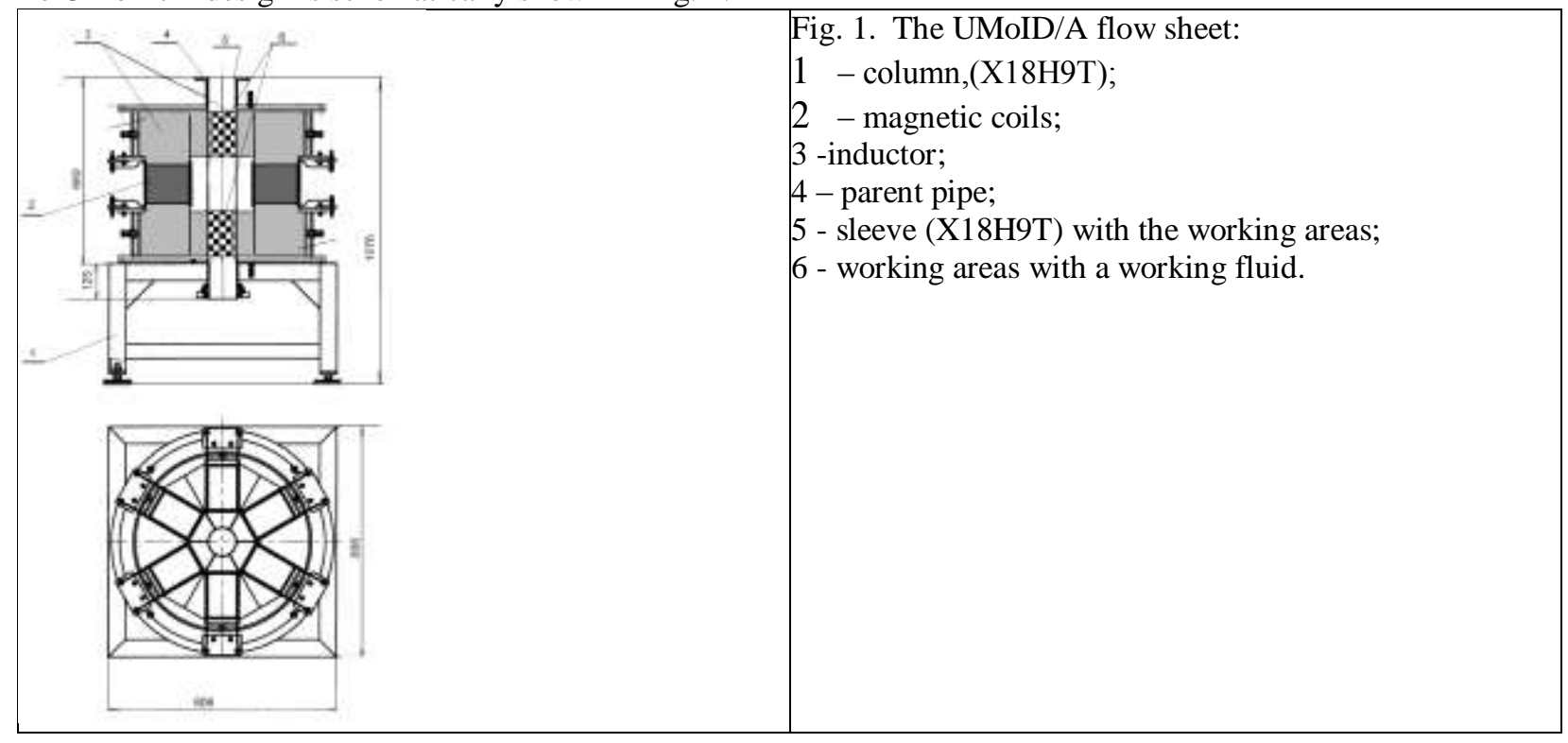

To increase productivity, modules should be installed in parallel. To obtain higher grinding parameters under constant productivity, the modules are connected in series. The authors showed that the profitability during grinding in UMM increases with increasing initial dispersion of the initial material. 
The schematic diagram of a continuous industrial grinding workshop is shown in Fig. 2. Fig. 3 shows a schematic sketch of a prototype installation for the production of materials using the UMoID/A-P on an industrial scale. The column of this device should allow you to change the slope of this module, providing the speed of the processed material passage through the module adjustment. Schematically, this device represents a serial connection of individual modules with two working areas also patented by the authors.

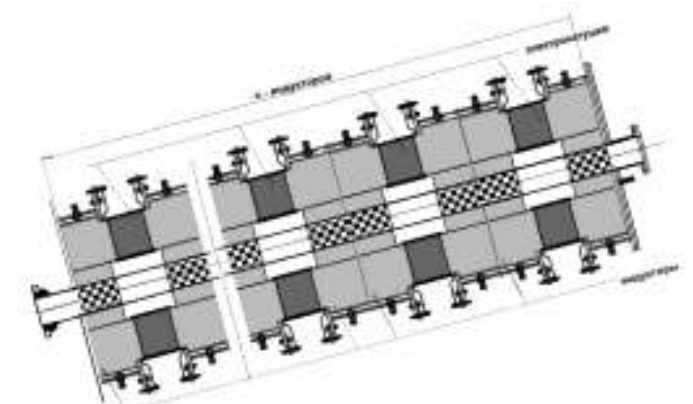

Fig. 2:- Schematic diagram of a continuous industrial grinding workshop: $\mathrm{M}$ - material pre-grinding mill; $\mathrm{UMoID} / \mathrm{A}-\mathrm{P}$ - continuous industrial material grinding modules.

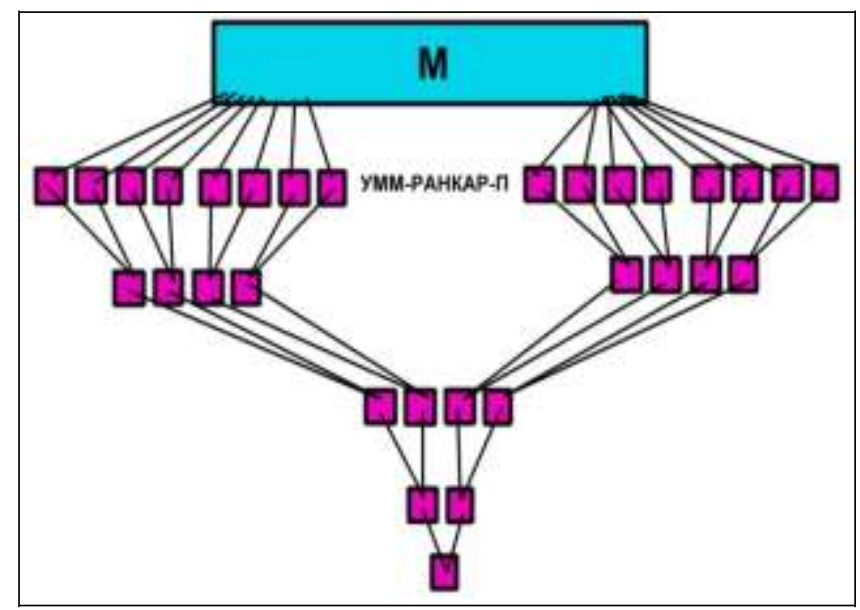

Fig. 3:- Schematic sketch of the UMoID/A-P prototype installation.

Preliminary calculations show that the economic efficiency of UMoID/A usage when grinding increases when the profitability of using other types of mills decreases. In the technologies discussed, the cost recovery is approximately 100 hours of the unit continuous operation. Depending on the technical problem being solved, the profitability of using new technologies can vary within the range from 100 to $500 \%$ and higher.

Based on the fact that carbon nanotubes have unique physical, chemical properties and are the most promising base material for creating various composite nanomaterials, the co-authors of this project have developed a very cheap method of natural K grade coal (anthracite coal) thermal modification.

The developed method of coal thermal modification includes several identical cycles of heating, soaking, hot pressing, cooling and final grinding (up to nanoscale) with a patented device - UMoID/A. A fine carbon powder is prepared from such a modified coal after its corresponding grinding in the UMMoID/A, mainly consisting of various types of carbon nanotubes (single-walled, multilayer, and telescopically embedded), and having ferromagnetic properties with a magnetic permeability of $\mu \sim 2$.

It should be noted that the cost of multilayer carbon nanotubes produced using the developed technology is only several times higher than the cost of the initial coal, and one processing plant can produce hundreds of tons of such modified coal, i.e., carbon nanoparticles per day. 
The carbon nanotubes production directly from natural coal will completely defuse the issue of providing enterprises for new nanocomposites production with initial nanomaterial.

The carbon nanotubes produced by this technology have all the necessary properties to develop highly efficient nanotechnologies for new composites production - the technology for producing them is cheap, productive, and the nanotube material produced has magnetic properties, $\mu \sim 2$, which make it possible to control the formation of nanostructures under the influence of electromagnetic fields.

In this work, we consider in detail one of the most important world food and environmental problems — the problem of zooplankton cultivation in the ocean. The technology of producing an oceanic reactor is the basis for the technology of zooplankton industrial cultivation. The underlying processes in this technology are:

1. Electrodynamic self-assembly of macroscopic fractal frames of carbon nanotubes in the sea water electrolyte column;

2. concentration and saturation with sunlight of the bioreactor entire volume;

3. zoo/biomass production for agriculture and medicine;

Under modern conditions of civilization development, associated with an unlimited increase in energy consumption, an infinitely increasing level of industrial environmental pollution and a rapid decrease in crop areas, renewable energy sources, for example, "biofuel", are becoming increasingly important. However, its production on an industrial scale leads to a sharp reduction in crop areas for food production and agricultural lands, since the yield of even the most promising crops (from the point of view of biofuel production) rarely exceeds $\mathbf{4 0}$ centners per hectare. From this point of view, the ocean can be a zoomass reactor with actually unlimited productivity (up to $10^{4} \mathbf{t} / \mathbf{h a}$ ), since the technology will allow to grow the crop in a layer up to $\sim \mathbf{5 0} \mathbf{~ m}$.

This is precisely the relevance of such a technology, the development of which should be the appropriate project. The relevance of such a project is that its implementation will inevitably contribute to the solution of another major environmental problem related to the problem of global climate warming, since the industrial cultivation of zoomass is associated with a significant consumption of carbon dioxide dissolved in ocean water and accumulated in the atmosphere.

This project is aimed at a comprehensive solution to the fundamental problems associated with the possibility of obtaining a renewable energy source, highly productive technologies development for feed, protein, biological additives and fertilizers cultivation, as well as an important environmental task related to the problem of global warming.

The solution of the above problems within the framework of such a project is based on the following basic principles identified and described:

only the micro-dust component with its quantum bonds can be responsible for frame structures observed in the ocean surface photographic images;

"carbon nanotubes" are the main block of frame structures;

frame structures spontaneously assembled under the conditions of electric current generation in the medium (gas or electrolyte medium);

"carbon nanotubes" and similar structures from other elements play a significant role in the formation of a micro-dust frame under abnormal atmospheric phenomena - tornadoes, waterspouts and hurricanes;

Topological features of the ocean skeletal structures research have shown that they: consist of:

individual coaxial tubular (CT) blocks in the form of vertically and/or horizontally floating cylinders tightly packed as thin capillaries (in the form of $\mathbf{3 r d}$ generation carbon nanotubes and fragments of frame structures of various generations) with straight-line radial spokes -

connect (due to the action of surface tension forces) individual blocks into a single network (as in a skeleton) covering the entire ocean surface (frame structures $\sim 10^{3} \mathrm{~km}$ ); 


\section{Show a tendency:}

1. to self-similarity (to breeding);

2. to an increase in observed block sizes with an increase in ocean surface heave;

3. to blocks emergence (during a storm) above the average level up to $\mathbf{2}$ meters, due to their partial filling with sea foam;

4. to upbuild coaxial-tubular blocks in the form of telescopically embedded cylinders (with a ratio of adjacent diameters equal to two) and with radial spokes-bracings.

According to these conclusions, the skeletal structures of the ocean are based on capillaries upbuilt with structureforming dust, like "carbon nanotubes" or similar ones, dust particles from other chemical elements or compounds, for example, $\mathrm{SiO} 2, \mathrm{Al} 2 \mathrm{O} 3, \mathrm{TiC}$, etc., which are under the influence of the Earth's electric field $(\sim 180 \mathrm{~V} / \mathrm{m})$ and atmospheric electricity, providing the current and field connection between themselves, are able to spontaneously upbuild a frame of powerful structural clouds in the form of a continuous nanodust network. Getting on the ocean surface, fragments of such a framework are flooded, but do not submerge, because its surface is very active and in sea water medium it adsorbs gases dissolved in water very intensively, acquiring floatability.

With increasing heave of the ocean surface, these frame structures do not withstand the impact of elements. They are broken and crushed into smaller fragments, which, like garbage, can fill the internal volumes of unbroken blocks. At each point of such an oceanic broth, there are three phases of substance matter: solid - nanodust frame, liquid - salt water medium and gaseous - bubbles and films of gas dissolved in water adsorbed on the skeleton surface. Therefore, at each point of such a medium, there are conditions when the surface tension forces have effect even under water. This leads to the fact that the frame blocks, filled with fragments of crushed blocks and blocks of previous generations, are tighten with elastic bundles by surface tension forces. The strength of skeletal structures of the ocean and its blocks is determined by how densely they are packed with fragments of frame structures that have fallen to the ocean surface and fragments of blocks of previous generations. These hardened individual blocks gently and flexibly (with the possibility of stretching, due to surface tension forces), as in the skeleton joints, are joined together into a single frame structure of the ocean, which, like a network, covers its entire surface and partially fills it in depth. This is where the ocean skeletal structures skeletal properties manifest themselves.

We briefly formulate the basics of hypotheses put forward here that describe the capillary model of the ocean skeletal structures:

1. skeletal structures of the ocean are based on structure-forming dust, the basic elements of which can be carbon nanotubes or similar particles from other elements supplied to the atmosphere by volcanoes and deserts;

2. under the influence of the Earth electric field and atmospheric electricity, such dust upbuilds frame structures of clouds that can lead to abnormal atmospheric phenomena or fall to the ocean surface;

3. creating a separation of three phases (solid, liquid and gaseous) on its surface, frame structures provide the action of surface tension forces (due to incomplete wetting) and the adhesion of individual blocks into a single network of skeletal structures of the ocean even under water;

4. frame structures, adsorbing air dissolved in water and partially filling with foam on their active surface, float to the surface, that is, they have non-zero floatability;

5. skeletal structures of the ocean and its blocks strength is determined by the fact that they are densely packed with fragments of frame structures that have fallen to the ocean surface and fragments of blocks of previous generations;

6. skeletal properties of the ocean skeletal structures is manifested in the fact that its rather rigid individual blocks flexibly and gently (with the possibility of stretching, due to surface tension forces) are joined together, as in a skeleton.

If under the influence of a flowing weak electric current in an electrolyte of sea water with carbon nanotubes create a volume of a vertically floating cylinder of fractal frame structures, which is the structure of the above capillaries from carbon nanotubes, partially filled with foam, then such a block will be afloat. This will happen under the condition that the buoyancy force caused by the gas adsorbed on the surface of capillaries (dissolved in water) and foam bubbles (stuck in the capillaries) will exceed the weight of the fractal frame structure together with the zoomass grown on it. The authors showed that the strength of such a block of the ocean skeletal structures is about $0.1 \div 0.5$ $\mathrm{kg} / \mathrm{cm}^{2}$. This allows easily separating the oceanic reactor active region from the open ocean. The versatility of the phenomenon of fractal frame structures and the predictive power of basic hypotheses defined for constructing and observed properties of the ocean skeletal structures makes it advisable to conduct detailed experimental and 
theoretical studies of kinetic processes that can lead to a formation of fractal frame structures with unique strength and productive capabilities for growing zooplankton on them in the oceanic environment.

\section{The main practical results of such a separate project will be:}

an oceanic reactor construction for highly efficient industrial production of zoo/bioplankton with a zoomass productivity of up to $\mathbf{1 0}^{4}$ t/ha per year) based on nanotechnology of electrodynamic self-assembly of macroscopic fractal frames from carbon nanotubes in the ocean layer;

development of technologies for zoo/biomass production and processing for the production of "biofuel", agriculture and medicine needs;

creation of a new industry for the industrial production of biomass.

\section{References:-}

1. V.A. Rantsev-Kartinov, A.V. Kulakov "Universal module of industrial disintegrators/activators", utility model patent No. 161751. Bulletin of the Federal Service for Intellectual Property, Patents, and Trademarks No. 13, dated 10.05.2016.

2. A.V. Kulakov, V.A. Rantsev-Kartinov Eurasian patent of invention No. 029979 "Device for a universal module of industrial disintegrators/activators" June 9, 2018.

3. A.V. Kulakov, V.A. Rantsev-Kartinov, V. M.Tyutuynnik New Technologies For Processing Coal Using Universal Module Industrial Disintegrators /Activators. American Journal of Engineering Research. 2018, Vol-7, Issue-11, pp-33-41.

4. A.V.Kulakov, V.A.Rantsev-Kartinov, V.M. Tyutyunnik Application of Universal Multipurpose Modules of Industrial Disintegrators /Activators for the Processing of Cereals and Potatoes into Starch Products. International Journal of Advanced Research Res. 5(5).1759-1762,(2017). 